\title{
Intervenção fisioterapêutica na incontinência fecal no idoso
}

\section{Physiotherapeutic intervention in fecal incontinence in elderly}

Lucas Lima Ferreira ${ }^{1}$, Laís Helena Carvalho Marino², Simone Cavenaghi²

\section{RESUMO}

O objetivo deste estudo foi agrupar e atualizar conhecimentos referentes à intervenção fisioterapêutica na incontinência fecal no idoso. A pesquisa da literatura foi realizada nas bases de dados eletrônicas: MEDLINE, LILACS, Cochrane, PubMed e SciELO, no período de janeiro de 2006 a julho de 2011. As palavras-chave usadas em várias combinações foram: fecal incontinence, aged, physical therapy, physiotherapy, exercises, rehabilitation e electrotherapy. A intervenção fisioterapêutica incluiu a avaliação da incontinência fecal constituída pelos sinais e sintomas, exame físico e exames complementares, que incluíram a manometria anorretal, a retossigmoidoscopia, a defecografia, a eletromiografia anorretal, a ultrassonografia endoanal e a latência motora terminal do nervo pudendo; os exercícios para fortalecimento da musculatura do assoalho pélvico, o biofeedback, o treinamento com balão retal e a eletroestimulação constituem a base do tratamento. Essa atualização demonstrou a escassez de estudos abordando tal temática, encontrando apenas cinco estudos publicados nos últimos anos com pacientes idosos incontinentes, os quais enfatizaram em sua maioria os procedimentos já consagrados na literatura refutando as evidências já conhecidas. Porém, uma modalidade consideravelmente nova de tratamento, a neuroestimulação sacral, tem sido investigada com resultados aparentemente positivos, suscitando a necessidade de novos estudos com desenhos metodológicos específicos sobre este problema.

Palavras-chave: incontinência fecal; idoso; fisioterapia.

\begin{abstract}
The objective of this study was to group or classify and update knowledge related to physical therapy intervention in fecal incontinence in the elderly. The literature search was conducted in electronic databases: MEDLINE, LILACS, Cochrane, PubMed and SciELO, from January 2006 to July 2011. The keywords used in various combinations were: fecal incontinence, aged, physical therapy, physiotherapy, exercises, electrotherapy and rehabilitation. The physiotherapy intervention includes assessment of fecal incontinence consisted by the signs and symptoms, physical examination and laboratory tests, including anorectal manometry, the sigmoidoscopy, the defecography, anorectal electromyography, ultrasonography and endoanal terminal motor latency of the pudendal nerve; strengthen exercises pelvic floor muscles, biofeedback, rectal balloon training and electrostimulation are the mainstay of treatment. This update showed the lack of studies addressing this issue and found only five studies published in recent years with elderly incontinent patients, which emphasized mostly the procedures already established in the literature refuting the evidence already known. However, a new treatment modality considerably, sacral neurostimulation has been investigated with apparently positive results, raising the need for new studies with methodological designs on this specific problem.
\end{abstract}

Keywords: fecal incontinence; aged; physical therapy specialty. 


\section{INTRODUÇÃO}

A Incontinência Fecal (IF) é o termo utilizado para englobar tanto a perda involuntária de material fecal quanto de gases, sendo caracterizada como a incapacidade para manter o controle fisiológico do conteúdo intestinal em local e tempo socialmente adequados, levando a perda involuntária de fezes líquidas, pastosas ou sólidas ${ }^{1-4}$. Essa alteração funcional pode gerar insegurança, perda da autoestima, angústia, depressão, transtornos físicos, mentais e sociais, que podem contribuir para uma piora na qualidade de vida dos indivíduos ${ }^{5,6}$.

A IF é infelizmente um problema social e médico com grande impacto socioeconômico: representa na América a segunda causa de hospitalização de idosos em instituições de longa permanência para idosos, com uma despesa de mais de \$ 400 mil dólares em fraldas e absorventes geriátricos ${ }^{7}$.

A prevalência exata dessa disfunção é desconhecida; porém, estima-se que 2 a $7 \%$ da população apresente algum grau de $\mathrm{IF}^{4,6,8}$. Esses dados podem não ser muito fidedignos, pois a prevalência da IF é subestimada pelo fato de ser sub-relatada, sendo que, muitas pessoas acometidas pela incontinência não procuram assistência dos profissionais de saúde, devido ao medo, frustração e vergonha ${ }^{6}$. Sabe-se, entretanto, que a incontinência é mais comum em idosos e no sexo feminino, provavelmente devido a fatores relacionados ao parto e à maior prevalência de constipação intestinal crônica nas mulheres ${ }^{9,10}$.

Entre os idosos, a ocorrência dessa disfunção pode atingir entre 17 a $47 \%$ e, na população brasileira, um estudo realizado no Ambulatório de Geriatria do Hospital das Clínicas de São Paulo encontrou prevalência de $10,9 \%^{4,11}$. Com o envelhecimento, ocorre degeneração do esfíncter anal interno, que pode causar redução progressiva das pressões de repouso, também pode ocorrer redução da complacência retal, da sensibilidade anal e atrofia muscular do assoalho pélvico, acarretando redução das pressões de contração ${ }^{11}$.

A fisioterapia é apontada como um dos procedimentos no tratamento dessa disfunção, por ser eficaz e poder evitar ou retardar o processo cirúrgico. No caso da IF, mediante a reeducação dos músculos do pavimento pélvico, exercícios específicos e eletroestimulação podem melhorar e diminuir as disfunções da estática lombopélvica, facilitando a função esfincteriana e promovendo até a correção de outras disfunções ${ }^{12-14}$.

Contudo, estudos com enfoque sobre a IF em populações não idosas têm sido descritos ${ }^{13,14}$; porém, trabalhos agrupando e sintetizando a abordagem da fisioterapia sobre pacientes idosos portadores de IF não foram encontrados na literatura atual. Nesse contexto, entende-se a importância de estudos que reúnam informações sobre o tema, haja vista que a incontinência, em todas as suas formas, é uma das patologias mais prevalentes sobre a população geriátrica.

Assim sendo, o objetivo deste estudo foi agrupar e atualizar conhecimentos referentes à intervenção fisioterapêutica na incontinência fecal no idoso.

\section{MÉTODO}

A pesquisa da literatura foi realizada nas bases de dados eletrônicas: MEDLINE, LILACS, Cochrane, PubMed e SciELO, no período de janeiro de 2006 a julho de 2011.

As palavras-chave usadas em várias combinações foram: $f e$ cal incontinence, aged, physical therapy, physiotherapy, exercises, rehabilitation e electrotherapy.

A pesquisa foi limitada às línguas inglesa, espanhola ou portuguesa, com estudos realizados em seres humanos adultos com 60 anos ou idade média superior a 60 anos de idade e que tinham sido publicados nos últimos cinco anos. As referências bibliográficas das pesquisas consultadas também foram usadas como levantamento de publicações referentes ao tema. Foi realizada uma análise de títulos e resumos para obtenção de artigos potencialmente relevantes para a revisão.

Com base nos critérios pré-estabelecidos para esta atualização, foram encontrados cinco artigos relevantes ao tema proposto, além de uma publicação referente a um protocolo de estudo com dois modelos de intervenção sobre a incontinência a ser testados, que serão apresentados a seguir com base em seus objetivos, metodologia, resultados e conclusões. Antes, porém, será abordado um capítulo relacionado à avaliação fisioterapêutica na IF como forma de nortear os profissionais da área sobre os principais focos da investigação dessa condição patológica.

\section{Avaliação fisioterapêutica na Incontinência Fecal}

A avaliação da IF inclui a avaliação clínica, constituída pelos sinais, sintomas e exame físico, além dos exames complementares, que incluem a manometria anorretal, a retosigmoidoscopia, a defecografia, a eletromiografia anorretal, a ultrassonografia endoanal e a latência motora terminal do nervo pudendo ${ }^{7,15}$.

O exame físico proctológico é constituído por inspeção estática, inspeção dinâmica, toque, anuscopia e retossigmoidoscopia e busca identificar anormalidades estruturais nos mecanismos de controle da defecação ${ }^{1,15}$. Na avaliação fisioterapêutica funcional, se inclui também a avaliação da capacidade de locomoção e posicionamento para defecar e as condições da musculatura pélvica estriada, tais como força, resistência, elasticidade e tonicidade muscular ${ }^{1,15}$.

A história clínica é geralmente focada nos sinais e sintomas que caracterizam o funcionamento intestinal, tais como: frequência e duração das perdas e das evacuações, consistência das fezes, presença de urgência, diarreia, flatulência e constipação e uso de proteção. Inclui ainda um relato da história cirúrgica e de doenças em outros sistemas que podem se relacionar com a ocorrência de incontinência, uso de medicações, hábitos alimentares, avaliação do estado mental e emocional ${ }^{1,15}$. Para uma melhor avaliação da IF, vários autores têm proposto um sistema de quantificação, que 
considere a intensidade e a frequência da perda, através de uma graduação numérica da gravidade ${ }^{15,16}$. Essa avaliação permite a comparação da efetividade das propostas terapêuticas entre pacientes, grupos e tipos de tratamento. As escalas Continence Grading Scale ou Escala de Classificação da Incontinência e Fecal Incontinence Severity Index (FISI) são as mais utilizadas nos ensaios clínicos ${ }^{17-19}$.

\section{Intervenção fisioterapêutica na Incontinência Fecal no idoso}

A intervenção fisioterapêutica da IF consiste em treinos para aumentar a capacidade contrátil e o controle voluntário do esfíncter anal externo e do elevador do ânus que respondem à distensão retal ${ }^{20}$. Desse modo, segundo Sobreira ${ }^{21}$, o tratamento fisioterapêutico de pacientes com IF tem como objetivos: melhorar a propriocepção vesical, retal e perineal; tonificar os músculos do assoalho pélvico, aumentando sua funcionabilidade, através da melhora da coordenação, força e resistência muscular e da sensibilidade anorretal; melhorar o reflexo de fechamento perineal ao esforço; melhorar o ciclo de continência - evacuação, além de melhorar a qualidade de vida $(\mathrm{QV})$.

Costa e Santos ${ }^{22}$ realizaram um estudo de casos com o objetivo de avaliar a eficiência do biofeedback e cinesioterapia na capacidade contrátil e propriocepção do assoalho pélvico em mulheres portadoras de IF. Foram avaliadas duas mulheres idosas de forma objetiva por intermédio do biofeedback e subjetiva por meio da Avaliação Funcional do Assoalho pélvico (AFA) e do Índice de Incontinência Anal (IIA), comparando os resultados antes e depois do tratamento. As pacientes realizaram o protocolo fisioterapêutico que constou de biofeedback e exercícios de Kegel na bola suíça. Ambas as pacientes realizaram 16 sessões, três vezes por semana. Os resultados mostraram melhora clínica das pacientes e ausência dos sintomas de IF.

Outro estudo de caso com paciente portador de IF teve como objetivo demonstrar que o biofeedback constitui um importante meio terapêutico para o tratamento da dissinergia do assoalho pélvico. Tratou-se do relato de um paciente, 62 anos, que apresentava IF e anismo e que realizou um tratamento com biofeedback. No total, foram realizadas dez sessões de biofeedback. Complementar às sessões, foi utilizado o método de cinesioterapia domiciliar, utilizando-se exercícios de contração e relaxamento do esfíncter anal similar aos realizados nas sessões de reeducação esfincteriana retoanal no consultório. O paciente apresentou grande evolução, com diminuição da dissinergia pélvica e da IF, melhora do controle esfincteriano e da QV. Assim, após a realização desse estudo, pode-se inferir que a associação do treinamento com biofeedback e a cinesioterapia domiciliar auxiliou no tratamento dessa enfermidade ${ }^{13}$.

Já um ensaio clínico prospectivo, realizado com o objetivo de analisar a capacidade de testes para prever o resultado da reabilitação do assoalho pélvico em pacientes com IF, foram selecionados 250 pacientes idosos submetidos à avaliação e testes específicos. Posteriormente, os pacientes foram encaminhados para reabilitação por meio de um protocolo que incluía treinamento com balão retal, estimulação elétrica e/ou feedback eletromiográfico. Os resultados da reabilitação foram quantificados pelo Índice de Incontinência Vaizey (IIV). Análises de regressão linear foram utilizadas para identificar candidatos preditores e para a construção de um modelo de previsão multivariada para a pontuação Vaizey pós-tratamento. O IIV no início do estudo foi de $18,3 \pm 3,0$. A média de pontuação Vaizey após a reabilitação foi de 15,0 $\pm 5,0$, uma redução média de 3,2 pontos (IC95\% -2,6--3,9; p<0,001). Testes adicionais têm um papel limitado na previsão de sucesso de reabilitação do assoalho pélvico em pacientes com $\mathrm{IF}^{23}$.

Em contrapartida aos estudos apresentados acima, nos quais foram utilizados métodos de tratamento da IF convencionalmente descritos pela literatura, Navarro et al. ${ }^{24}$ relataram a experiência inicial com uma técnica recente denominada neuroestimulação percutânea perissacral (NPS), em 26 pacientes idosos onde escores basais da Escala Wexner-Cleveland (EWC) e capacidade de adiar a defecação foram comparados com os resultados após um ano com NPS. Antes da NPS, a média de pontuação da EWC foi $15,00 \pm 1,81$ e $62,5 \%$ dos pacientes só podiam retardar a evacuação menos de um minuto. Depois de um ano com NPS, a pontuação média da escala foi de $4,87 \pm 2,54$ $(p=0,0031)$, e 75,01\% dos pacientes retardaram a defecação acima de $15 \mathrm{~min}(\mathrm{p}=0,0018)$. A NPS é uma técnica eficaz para o tratamento de IF em pacientes adequadamente selecionados, sem resposta a terapias médicas (incluindo biofeedback) ou à correção anatômica (esfincteroplastia), com eficácia, pouca morbidade e um tratamento de curta duração.

Outro estudo realizado com a NPS objetivou investigar se a técnica poderia melhorar pacientes com distúrbios de motilidade intestinal, com incontinência resistente durante seis anos em 19 pacientes ${ }^{25}$. A pesquisa demonstrou que a continência melhorou em todos os 24 meses (variação de 30-60 meses), 14 pacientes tornaram-se completamente continentes. Os episódios de incontinência diminuíram $(\mathrm{p}<0,001)$, urgência e QV melhoraram $(\mathrm{p}<0,05)$. A pressão de contração voluntária anal $(\mathrm{p}=0,001)$ e a sensação retal $(\mathrm{p}<0,01)$ melhoraram $^{25}$. Essa pesquisa fornece fortes evidências de que a NPS pode melhorar o quadro de pacientes com incontinência resistente e pode ser utilizada como coadjuvante para o tratamento da constipação.

Diferentemente dos estudos encontrados, Bols et al. ${ }^{26}$ publicaram um protocolo de estudo antes de obter resultados, para investigar a eficácia de duas intervenções de fisioterapia: treinamento com balão retal (TBR) e treinamento muscular do assoalho pélvico (TMAP) na gestão da IF. Tratou-se de um ensaio clínico randomizado controlado com 106 pacientes que irão receber TMAP combinado com TBR ou TMAP sozinho. As medidas de base consistiram no IIV, na história clínica, exame físico, uso de medicamentos, exames complementares, diário 
de sintomas, escala de QV em Incontinência Fecal (FIQL) e um escore de avaliação da severidade da incontinência fecal (PREFAB). As avaliações serão programadas em três, seis e 12 meses após a inclusão. Doze sessões de fisioterapia serão administradas durante três meses, de acordo com um protocolo padronizado. O TMAP consistirá na coleta seletiva de contrações voluntárias e relaxamento dos músculos do assoalho pélvico e do esfíncter anal. Já o TBR consistirá de um balão ligado a uma seringa, introduzido no reto e lentamente insuflado com ar para imitar conteúdo retal, onde o limiar sensorial, a sensação de urgência e o volume máximo tolerado são avaliados. A hipótese é que pacientes que receberem o treinamento combinado terão uma redução maior na medida de desfecho primário.

\section{CONSIDERAÇÕES FINAIS}

A incontinência fecal no idoso constitui grave disfunção, atingindo os portadores nos campos físico, social e emocional, devendo, portanto, ser enfatizada uma abordagem multidisciplinar sobre esses pacientes. Neste contexto, a intervenção fisioterapêutica se faz essencial e imprescindível, uma vez que esta pode favorecer a melhora desta disfunção, inibindo a necessidade de intervenção cirúrgica.

Apesar de esta afirmação ser compartilhada entre a comunidade médico-científica, essa atualização demonstrou a escassez de estudos abordando tal temática, encontrando apenas cinco estudos publicados nos últimos anos, os quais enfatizam em sua maioria os procedimentos já consagrados na literatura, cinesioterapia do assoalho pélvico e biofeedback, no tratamento desses pacientes, sem, contudo, apontar novas evidências. Porém, uma modalidade consideravelmente nova de tratamento, a neuromodulação através de eletroestimulação percutânea perissacral ou parassacral, tem sido investigada com resultados aparentemente positivos, suscitando à necessidade de novos estudos com desenhos metodológicos específicos sobre este problema.

\section{REFERÊNCIAS}

1. Cooper Z, Rose S. Fecal incontinence: a clinical approach. Mt Sinai J Med. 2000;67(2):96-105.

2. Rockwood T. Incontinence severity and QOL scales for fecal incontinence. Gastroenterology. 2004;126(Suppl.1):S106-13.

3. Kalantar JS, Howell S, Talley NJ. Prevalence of faecal incontinence and associated risk factors: an underdiagnosed problem in the Australian community? Med J Aust. 2002;176(2):54-7.

4. Barbosa JMM, Dias RC, Pereira LSM. Qualidade de vida e estratégias de enfrentamento em idosos com incontinência fecal: uma revisão da literatura. Rev Bras Geriatr Gerontol. 2007;10(3):383-99.

5. Oliveira SCM, Pinto-Neto AM, Conde DM, Góes JRN, Santos-Sá D, Costa-Paiva L. Incontinência fecal em mulheres na pós-menopausa: prevalência, intensidade e fatores associados. Arq Gastroenterol. 2006:43(2):102-6.

6. Quintão MG, Oliveira SAS, Guedes HM. Incontinência fecal: perfil dos idosos residentes na cidade de Rio Piracicaba, MG. Rev Bras Geriatr Gerontol. 2010;13(2):191-201.

7. Oliveira L. Incontinência fecal.J Bras Gastroenterol. 2006;6(1):35-7.

8. Johansen JF, Lafferty J. Epidemiology of fecal incontinence: the silent affliction. Am J Gastroenterol. 1996;91(1):33-6.

9. Oliveira L, Neves-Jorge JM, Yusuf S, Habr-Gama A, Kiss D, Cecconelo I. Novos tratamentos para a incontinência anal: injeção de silicone melhora a qualidade de vida em 35 pacientes incontinentes. Rev Bras Coloproct. 2007;27(2):167-73

10. Kok ALM, Voorhost FJ, Burger CW, Van Houten P, Kenemans P, Janssens $\mathrm{J}$. Urinary and fecal incontinence in community-residing elderly women. Age ageing. 1992;21(3):211-5.

11. Wald A. Constipation and fecal incontinence in the elderly. Gastroenterol Clin North Am. 1990;19(2):405-18.

12. Mancuso JP. Fisioterapia minimiza sintomas de incontinência fecal. [internet] Disponivel em: 〈http://www2.uol.com.br/vyaestelar/ fisioterapia_incontinencia.htm >. Acesso em: 11 set. 2011.
13. Arend MCP, Fernandes WVB, Arend G. Uso do biofeedback na incontinência fecal e dissinergia do assoalho pélvico: relato de caso. Rev Saude Pesq. 2009;2(3):433-6.

14. Netinho JG, Dourado HM, Starling F, Ramalho EN. Tratamento da incontinência anal com biofeedback. Resultados preliminares. Rev Bras Coloproctol. 1999;19(2):88-93.

15. Barbosa JMM. Avaliação da qualidade de vida e das estratégias de enfrentamento em idosos com incontinência fecal [dissertação]. Belo Horizonte: Escola de Educação Física, Fisioterapia e Terapia Ocupacional, Universidade Federal de Minas Gerais; 2006.

16. Perry S, Shaw C, McGrother C, Matthews RJ, Assassa RP, Dallosso H, et al. Prevalence of faecal incontinence in adults aged 40 years or more living in the community. Gut. 2002;50(4):480-4.

17. Yusuf SAl, Jorge JMN, Habr-Gama A, Kiss DR, Rodrigues JG. Avaliação da qualidade de vida na incontinência anal: validação do questionário FIQL (Fecal Incontinence Quality of Life). Arq Gastroenterol. 2004; $41(3): 202-8$

18. Rothbarth J, Bemelman W, Meijerink WJHJ, Stiggelbout AM, Zwinderman $\mathrm{AH}$, Buyze-Westerweel ME, et al. What is the impact of fecal incontinence on quality of life? Dis Colon Rectum. 2001;44(1):67-71

19. Damon H, Dumas P, Mion F. Impact of anal incontinence and chronic constipation on quality of life. Gastroenterol Clin Biol. 2004;28(1):16-20

20. Cheung 0 , Wald A. Review article: the management of pelvic floor disorders. Aliment Pharmacol Ther. 2004;19(5):481-95.

21. Sobreira MM.Aplicação da fisioterapia na reabilitação das disfunções do assoalho pélvico. In: Regadas SP, Regadas SMM. Distúrbios funcionais do assoalho pélvico. Atlas de ultra-sonografia anorretal bi e tridimensional. Rio de Janeiro: Revinter; 2007. p. 125-36.

22. Costa AP, Santos JG. Estudo prospectivo da resposta terapêutica ao biofeedback e cinesioterapia em pacientes portadoras de incontinência anal: relato de dois casos [monografia]. Belém: Universidade da Amazônia; 2008. 
23. Terra MP, Deutekom M, Dobben AC, Baeten CGMI, Janssen LVVM, Boeckxstaens GEE,et al.Can the outcome of pelvic-floor rehabilitation in patients with fecal incontinence be predicted? Int J Colorectal Dis. 2008:23(5):503-11.

24. Navarro JM, Arroyo Sebastián A, Pérez VF, Sánchez Romero AM, Pérez Legaz J, Serrano Paz P, et al. Sacral root neuromodulation as treatment for fecal incontinence. Preliminary results. Rev Esp Enferm Dig. 2007;99(11):636-42.

25. Kenefick NJ. Sacral nerve neuromodulation for the treatment of lower bowel motility disorders. Ann R Coll Surg Engl. 2006;88(7):617-23.
26. Bols EMJ, Berghmans BCM, Hendriks EJM, Bie RA, Melenhorst J, Gemert WC, et al. A randomized physiotherapy trial in patients with fecal incontinence: design of the PhysioFIT-study. BMC Public Health. $2007 \cdot 7 \cdot 355$

\section{Endereço para correspondência}

Lucas Lima Ferreira - Rua Gabriel Otávio de Souza, 855, apto. 24 -

Jardim Paulista - CEP: 19023-230 - Presidente Prudente (SP), Brasil Email:lucas_lim21@hotmail.com

Conflito de interesse: nada a declarar. 